\title{
РАННИЙ ПОСТГАСТРОРЕЗЕКЦИОННЫЙ СИНДРОМ
}

\section{EARLY POSTGASTRECTOMY SYNDROME}

A. Zorkin

Summary. The article considers the course of the early postoperative period after performing distal gastrectomy in complicated chronic gastroduodenal ulcers in 84 patients. The frequency and duration of maintaining the symptom complex of organ lesion of the operated stomach, designated as early postgastrectomy syndrome (EPGES), are considered. It is noted that at an incidence rate of about $70 \%$, these clinical manifestations are non-specific and statistically significantly more common after gastrectomy performed by one of the modifications of Billroth-2 than Billroth-1. The duration of EPGES is more than 5 days, and the severe course requires the exclusion of postoperative complications in order to perform a timely repeated surgical intervention.

Keywords: gastroduodenal peptic ulcer, surgical treatment, gastrectomy, postgastrectomy diseases.

\author{
Зорькин Алексей Александрович \\ К.м.н., дочент, БУВО ХМАО-Югры «Сургутский \\ государственный университет» \\ az_99@mail.ru
}

Аннотация. В статье рассматриваются вопросы течения раннего послеоперационного периода после выполнения дистальной резекции желудка по поводу осложнений хронических гастродуоденальных язв у 84 пациентов. Рассмотрена частота и длительность сохранения симптомокомплекса органного поражения оперированного желудка, обозначенного, как ранний постгастрорезекционный синдром (РПГРС). Отмечено, что при частоте возникновения около 70\%, данные клинические проявления неспецифичны и статистически значимо чаще встречаются после резекции желудка, выполненной по одной из модификаций Бильрот-2, чем по Бильрот-1. Длительность РПГРС более 5 суток, и тяжелое течение требует исключения послеоперационных осложнений для выполнения своевременного повторного оперативного вмешательства.

Ключевые слова: язвенная болезнь желудка и двенадцатиперстной кишки, хирургическое лечение, резекция желудка, болезни оперированного желудка.

ний набольшее внимание уделяется несостоятельности швов и кровотечению в просвет желудочно-кишечного тракта в области анастомоза, а другие менее грозные симптомы чаще относят за счет послеоперационного пареза кишечника $[3,7,8,10,12,18]$.

\section{Цель исслеАования}

Изучить ряд болезненных проявлений в раннем послеоперационном периоде, связанных с радикальными операциями по поводу перфоративных и кровоточащих хронических язв желудка и двенадцатиперстной кишки.

\section{Материалы и метомы}

В проспективное исследование включены 84 выздоровевших пациента, которые перенесли дистальные резекции желудка в различных модификациях, в связи с остро возникшими осложнениями язвенной болезни желудка и двенадцатиперстной кишки (ЯБЖ и ЯБДПК). Отбор пациентов проводился в течение периода 20002020 г.г. в хирургических отделениях МУЗ «Городская больница № 1» г. Барнаула и БУ «Сургутская городская клиническая больница». Подход к критериям включения, исключения и невключения был единым. Критериями включения было наличие у пациента перфорации хронической язвы желудка или ДПК, потребовавшего экстренной операции продолжающегося гастродуоде- 
Таблица 1. Частота регистрации РПГРС

\begin{tabular}{|l|l|l|l|l|l|l|}
\hline & \multicolumn{4}{|l|}{ Резекция по Бильрот-1 } & \multicolumn{3}{l|}{ Резекция по Бильрот-2 (модификации) } \\
\hline Степень & Легкая & Средняя & Тяжелая & Легкая & Средняя & Тяжелая \\
\hline ЯБДПК & $6(15,8 \%)$ & $3(7,9 \%)$ & 0 & $20(43,4 \%)$ & $7(15,2 \%)$ & $3(6,5 \%)$ \\
\hline ЯБЖ & $7(18,4 \%)$ & $3(7,9 \%)$ & $2(5,3 \%)$ & $2(4,3 \%)$ & $3(6,5 \%)$ & $1(2,2 \%)$ \\
\hline Всего & $13(34,2 \%)$ & $6(15,8 \%)$ & $2(5,3 \%)$ & $22(47,7 \%)$ & $10(21,7 \%)$ & $4(8,7 \%)$ \\
\hline
\end{tabular}

Примечание. Указано количество больных и доля среди оперированных по методике.

нального кровотечения язвенной этиологии, а также сочетания этих осложнений, в т.ч. с пенетрацией и стенозом выходного отдела желудка или ДПК, по поводу которых была выполнена экстренная или срочная дистальная резекция желудка. Критериями невключения были терминальное состояние больного, наличие признаков рефрактерного к терапии геморрагического или септического шока, полиорганной недостаточности, распространенного перитонита, возраст более 80 лет, наличие декомпенсированной сопутствующей патологии, проводимая терапия кортикостероидами и НПВС, выполнение органосохраняющего вмешательства. Критериями исключения были неблагоприятный исход заболевания, потребность в назначении кортикостероидной терапии и НПВС в первые 7 суток послеоперационного периода. В качестве двух контрольных групп отобрано по 30 оперированных по поводу:

1. острого аппендицита без перитонита (операция - аппендэктомия, видеолапароскопическая аппендэктомия),

2. перфораций острых и хронических язв желудка и ДПК (операция - открытое или видеолапароскопическое ушивание перфоративного отверстия, санация, дренирование брюшной полости).

Группы были сопоставимы по основным эпидемиологическим и клиническим параметрам: полу, возрасту, проявлениям сопутствующей патологии. Изучали частоту и длительность органных проявлений поражения культи желудка по общеклиническим данным, при необходимости проводили эндоскопическую и рентгенологическую диагностику нарушений эвакуаторной функции культи желудка. Оценку проводили методами вариационной статистики с использованием пакета прикладных программ Statistica 6.1 с учетом характера распределения показателей. Различия показателей считали статистически значимыми при критическом уровне значимости (р) менее 0,05.

\section{Результаты и их обсужление}

у 27 больных (32,1\%) тяжесть состояния определялась объемом вмешательства, выраженностью осложнений основного заболевания: перитонит, анемия, водно-электролитные нарушения, абдоминальный сепсис, почечная недостаточность, сопутствующей сердечно-легочной патологией. Симптомов, напрямую связанных с операцией на желудке и дПК, не было выявлено. В 57 случаях (67,9\%) дополнительно имелись признаки органного поражения оперированного желудка: тошнота, икота, рецидивирующая рвота в сочетании с чувством тяжести в эпигастрии, объективно отмечалась выполненность в подложечной области. Пальпация ее усиливала тошноту, иногда провоцировала рвоту. Из культи желудка удалялось значительное количество застойного содержимого. Данный симптомокомплекс, обозначенный как ранний постгастрорезекционный синдром (РПГРС) наблюдался у пациентов любого пола и возраста, локализации язвы (желудок, ДПК), при любой распространенности процесса на окружающие ткани, любой методике резекции желудка (по Бильрот-1, по Бильрот-2 в модификациях Гофмейстера-Финстерера, Витебского, Бальфура и др.), при всех видах осложнений основного заболевания. Различной была только степень выраженности вышеуказанных признаков:

1. Легкая - не причиняющая значительных страданий тошнота, одно-двухкратная необильная рвота. Пальпация области эпигастрия рвоту не провоцирует. При постановке зонда в культю желудка количество застойного отделяемого не превышает 500 мл.

2. Средняя - тошнота, срыгивание застойного желудочного содержимого либо многократная необильная рвота. При пальпации эпигастрия усиливается тошнота. Из культи желудка эвакуируется не менее 1 литра застойного отделяемого.

3. Тяжелая - сильная тошнота, икота, многократная обильная рвота «полным ртом». В эпигастрии отмечается выбухание, иногда ассиметрня, как правило на фоне мышечной защиты. Пальпация этой области в большинстве случаев провоцирует рвоту. Из культи желудка по зонду одномоментно удаляется не менее 1,5 литров, а суточные потери составляют не менее 3 литров застойного содержимого.

Распределение симптомокомплекса по степени тяжести в зависимости от локализации язвы и методики операции представлено в таблице 1. 
Таблица 2. Длительность регистрации РПГРС

\begin{tabular}{|l|l|l|l|l|l|l|}
\hline & \multicolumn{2}{|l|}{ Резекция по Бильрот-1 } & \multicolumn{3}{l|}{ Резекция по Бильрот-2 (модификации) } \\
\hline Степень & Легкая & Средняя & Тяжелая & Легкая & Средняя & Тяжелая \\
\hline $1-3$ суток & $12(31,6 \%)$ & $1(2,6 \%)$ & 0 & $21(45,6 \%)$ & $2(4,4 \%)$ & 0 \\
\hline 3-5 суток & $1(2,6 \%)$ & $2(5,2 \%)$ & 0 & $1(2,2 \%)$ & $7(15,2 \%)$ & $2(4,4 \%)$ \\
\hline Более 5 суток & 0 & $3(7,9 \%)$ & $2(5,2 \%)$ & 0 & $1(2,2 \%)$ & $2(4,4 \%)$ \\
\hline
\end{tabular}

Примечание. Указано количество больных и доля среди оперированных по методике.

Признаки органного поражения оперированного желудка имелись у 21 пациента из 38 после резекции желудка по Бильрот-1(55,3\%), а при резекции по одной из модификаций Бильрот-2 -у 36 больных из 46(78,26\%), $\mathrm{p}<0,05$. Среднетяжелая и тяжелая его степень была встречалась чаще после операций по Бильрот - 2 (7 из 38, 21,1\% и 14 из 46, 30,4\%, p<0,05), хотя в распределении внутри групп, объединенных по способу резекции, отмечены несущественные различия.

В таблице 2 представлены сроки исчезновения симптомокомплекса РПГРС при различной степени его выраженности в зависимости от способа резекции.

Таким образом, при легкой степени проявлений их продолжительность не превышает 5 суток, а в более 90\% наблюдений - 3 суток вне зависимости от способа резекции, при средней - в срок до 5 суток клиника исчезает у 75\% пациентов (у 7\% - до 3 суток), при резекции по Бильрот-2 быстрее, чем после операции по Бильрот-1 $(p<0,05)$. При тяжелой степени выраженности симптомов только в трети случаев продолжительность их была менее 5 дней. Из 4 больных, у которых свыше 5 дней имелись признаки органного поражения оперированного желудка, 1 был оперирован повторно в связи с перитонитом, развившимся в результате несостоятельности швов анастомоза. У 3 пациентов эндоскопически и рентгенологически диагностирован анастомозит с тотальным или субтотальным блоком пассажа через зону гастроэнтероанастомоза. Таким образом, длительность РПГРС более 5 суток подразумевает абсолютную морфологическую причину нарушений, требующую оперативной или эндоскопической коррекции.

Изучение групп контроля показало отсутствие указанных выше признаков у всех больных, оперированных по поводу острого аппендицита. Двое пациентов с ушитыми перфоративными язвами ДПК в течение 2 суток после операции отмечали тошноту, у одного была однократная необильная рвота, т.е. в 6,7\% случаев имелись нарушения легкой степени.

Очевидно, указанный симптомокомплекс, возникающий после радикальных вмешательств на желудке и ДПК в раннем послеоперационном периоде у больных со сходной патологией, неспецифичен и имеет единый патогенетический механизм. Основой его могут быть функциональные нарушения, приводящие к разобщению секреторно-экскреторной и двигательной активности культи желудка. В условиях острой локальной травмы происходит дискоординация нейрогенных и гуморальных механизмов регуляции желудочных функций с относительным преобладанием секреции и застоем содержимого в культе желудка $[5,11]$. Исследованиями А.А. Курыгина показано, что в ранние сроки после резекции желудка происходит выраженное и стойкое угнетение сократительной активности его культи [10]. Адинамия культи выявляется у 75\%. гиподинамия - у 25\%. Потенцирует эти механизмы посттравматический панкреатит, в ряде случаев возникающий чаще после операций по Бильрот-2 в связи с манипуляциями на поджелудочной железе при выделении пенетрата $[6,12,18]$. Клинические исследования показывают, что в этих условиях более, чем у $80 \%$ больных имеется гиперхлоргидрический или гиперреактивный типы секреции соляной кислоты, при которых эффективность резекций желудка ниже, чем при гиперантральном типе $[16,17,20]$. В большинстве случаев сохраняющаяся относительная гиперсекреция после операции в сочетании с денервацией части культи желудка, области анастомоза, его травматическим отеком, рефлюксом желчи ведет к описанным выше клиническим проявлениям. Присутствуют гемодинамические расстройства, в том числе и локальные, нарушения реологических свойств крови, различная степень дегидратации и гипоксии $[10,12]$.

Таким образом, можно говорить о наличии у больных, перенесших радикальную операцию на желудке и ДПК по поводу осложненной язвенной болезни, раннего постгастрорезекционного синдрома (РПГРС). Опасность его развития и прогрессирования очевидна. Накопление большого количества содержимого в культе желудка создает предпосылки для:

1. Несостоятельности швов анастомоза.

2. Кровотечения из зоны анастомоза.

3. Образования острых язв и эрозий культи желудка.

4. Развития анастомозита. 
Имеющиеся водно-электролитные нарушения в ходе развития РПГРС, особенно тяжелой степени, усугубляются и требуют длительной и продуманной программы интенсивной терапии, прежде всего инфузионио-трансфузионной (ИТТ). Первостепенное значение имеет поддержание ОЦК и степени гидратации тканей, электролитных параметров сред организма, формирование положительного энергетического и метаболического баланса. Данные литературы отмечают эффективность мероприятий, обеспечивающих удовлетворительные условия гемоциркуляции и оксигенации как в целом, так и непосредственно в области операции (вспомогательная вентиляция легких, гипербарическая оксигенация, улучшение реологии крови и борьба с скрытым ДВС-синдромом, гемотрансфузии по показаниям, ранняя и длительная декомпрессия культи резецированного желудка, применение методов экстракорпоральной гемокоррекции и пр.)[1, 12, 15]. Современные комплексные подходы в ведении послеоперационного периода включают раннюю активизацию больного и возможно более ранний перевод его на частичное или полное энтеральное питание[13, 14, 15, 19]. Наличие РПГРС накладывает значительные ограничения на эту тактику, вынуждая проводить длительную и многокомпонентную ИТТ с элементами парентерального питания, что не может не отразиться на эффективности и стоимости лечения.

Прогностическое значение РПГРС не менее важно. Наблюдая картину тяжелого, а особенно тяжелого и длительного его течения, с трудом поддающегося терапии, необходимо предположить наличие осложнения не функциональной, а более материальной природы (несостоятельность швов анастомоза, анастомозит, ранняя спаечная кишечная непроходимость, послеоперационный панкреатит). В этом случае проведение комплекса диагностических мероприятий, включая рентгенологические и эндоскопические, не должно затягивать выставление показаний к релапаротомии. Только своевременность выявления и хирургической коррекции послеоперационных осложнений может быть необхо- димым условием успешного выздоровления пациента и снижения показателей летальности при осложненном течении язвенной болезни.

\section{Выво $\triangle ы$}

· У 67,9\% больных, перенесших резецирующую операцию по поводу осложнений язвенной болезни желудка и ДПК, в ранние сроки после операции регистрируется симтомокомплекс органного поражения оперированного желудка, обозначенный как ранний постгастрорезекционный синдром (РПГРС).

- Клинические проявления РПГРС неспецифичны и на зависят от выбранных хирургом объема и модификации резекции желудка.

. РПГРС статистически значимо чаще встречается после резекции желудка, выполненной по одной из модификаций Бильрот-2, чем по Бильрот-1, в целом - на 23\%, среднетяжелые и тяжелые формы - на 9,3\%.

- Длительность РПГРС при легкой степени проявлений не превышает 5 суток, а в более $90 \%$ наблюдений 3 суток и не зависит от способа резекции; при средней степени у 3/4 больных не превышает 5 суток, при резекции по Бильрот-2 проявления купируются быстрее, чем после операции по Бильрот-1; при тяжелой степени у более 2/3 больных длительность превышает 5 суток вне зависимости от способа резекции.

- Длительное, более 5 суток, и тяжелое течение РПГРС требует исключения послеоперационных осложнений, требующих повторного оперативного вмешательства.

Более глубокая и детальная разработка вопросов патогенеза, диагностики и методов комплексного лечения РПГРС позволит улучшить ближайшие и отдаленные результаты радикального оперативного лечения осложненных форм ЯБЖ и ЯБДПК, повысить эффективность интенсивной терапии в раннем послеоперационном периоде у этой группы больных.

\section{ЛИТЕРАТУРА}

1. Афендулов, С. А. Стратегия хирургического лечения язвенной болезни./ С. А. Афендулов, Г. Ю. Журавлев, А. Д. Смирнов. // Хирургия.— 2006.— № 5.— C. $26-30$.

2. Белоногов, Н.И. Осложнения язвенной болезни желудка и двенадцатиперстной кишки и их хирургическое лечение. / Н. И. Белоногов, Е. Н. Валыка, В. В. Янголенко. // Мед. альманах. — 2011. — № 2 (15). — С. 68-71.

3. Борисов, А.Е. Неотложная хирургия язвенной болезни желудка и двенадцатиперстной кишки./ А. Е. Борисов, В. П. Земляной, В. П. Акимов, А. К. Рыбкин.-СПб.: Человек, 2002. - 48 с.

4. Гришаева, А. С. Хирургическое лечение сочетанных осложнений язв желудка и двенадцатиперстной кишки. / А. С. Гришаева, А. А. Полежаев, В. И. Макаров и др.// Материалы ХІ съезда хирургов Российской Федерации. - Волгоград. - 2011. - C. 660-661.

5. Евсеев, М. А. Антисекреторные препараты в неотложной хирургической гастроэнтерологии. / М. А. Евсеев.— М.: «КВАН».— 2009. — 173 c. 
6. Жанталинова, Н.А. Выбор хирургической тактики при гигантских язвах желудка и двенадцатиперстной кишки./ Н. А. Жанталинова // Хирургия.2005.—№ 12. - C. 30-32.

7. Жигаев, Г.Ф. Постгастрорезекционные осложнения язвенной болезни./ Г.Ф.Жигаев, Е. В. Кривигина.// Сибирский медицинский журнал.- 2010.№ $6 .-$ - $.35-36$.

8. Захарова, Г. Н. Хирургия желудка./ Г. Н. Захарова, В. Ф. Чуенков.- Саратов, Изд-во Саратовского университета, 1971.- 185 С.

9. Ивашкин, В. Т. Рекомендации по диагностике и лечению язвенной болезни (пособие для врачей)./ В. Т. Ивашкин, А. А. Шептулин, Е. К. Баранская и др.M., 2005.- 30 c.

10. Курыгин, А. А. Моторная и эвакуаторная функция пищеварительного тракта после операций на желудке (клинико-экспериментальное исследование).: автореферат дис. ... доктора медицинских наук.: 14.00.27. — Санкт-Петербург, 1997. — 44 с.: ил.

11. Мыш, Г. Д. Секреторная функция оперированного желудка./ Г. Д. Мыш.// Клин.хирургия. — 1982.— № 6._ С. 24-26.

12. Неймарк, И. И. Прободная язва желудка и двенадцатиперстной кишки./ И. И. Неймарк. - Барнаул, Алт.кн.изд-во, 1988. — 336 с.

13. Никитин, Н. А. Хирургическое лечение сочетанных осложнений язвенной болезни при пилородуоденальной локализации язв. / Н. А. Никитин, Т. П. Коршунова, М. А. Онучин, А. А. Головизнин. // Медицинский альманах.— 2010.— № 1 (10). — C. 121-125.

14. Пасечник, И.Н.Программа ускоренного выздоровления: особенности взаимодействия хирурга и анестезиолога-реаниматолога./ И. Н. Пасечник, Г. Н. Хрыков.// Альманах института хирургии им. А. В. Вишневского. — 2017. — № 1. - С. 377-379.

15. Попова, Т. С. Нутритивная поддержка больных в критических состояниях./ Т. С. Попова, А. Е. Шестопалов, Т. Ш. Тамазошвили, И. Н. Лейдерман. - М.: Вестник. - 2002. - $320 \mathrm{C}$.

16. Рыбачков, В.В.Язвенная болезнь желудка и двенадцатиперстной кишки./ В. В. Рыбачков, Р. М. Евтихов, И.Г. Дряженков, М. И. Сим.- Ярославль: 000 «Издательский дом «Верхняя Волга».— 2008. - 272 с.

17. Скляров, Е. Я. Факторы агрессии и защиты при пептической язве желудка и двенадцатиперстной кишки./ Е. Я. Скляров, И. Н. Тумак, Е. Е. Склярова.// Российский журнал гастроэнтерологии, гепатологии, колопроктологии.— 2012.— № 5.— Прил. 40.— С. 40.

18. Тарасенко, С. В. Хирургическое лечение «трудных» язв двенадцатиперстной кишки. / С. В. Тарасенко, О. В. Зайцев, О. Д. Песков, А. А. Копейкин. // Хирургия.—-2005. - № 1.—C. 29-32.

19. Толстокоров, А. С. Особенности хирургического лечения больных с сочетанными осложнениями язвы желудка и двенадцатиперстной кишки./ А. С. Толстокоров, С. А. Дергунова, Р. В. Тимохин, А. Д. Бахаев // В сб.: Новые технологии в хирургии и интенсивной терапии. — Саранск, 2010.— С. 243-244.

20. Хачиев, Л. Г. Результаты хирургического лечения язвенной болезни в зависимости от кислотопродуцирующей функции желудка. / Л. Г. Хачиев, Ю. И. Калиш, И. М. Постолов // Хирургия.— 1985.—№ 10.—C. 29-33.

( ) Зорькин Алексей Александрович ( az_99@mail.ru ).

Журнал «Современная наука: актуальные проблемы теории и практики» 